Provided for non-commercial research and education use. Not for reproduction, distribution or commercial use.

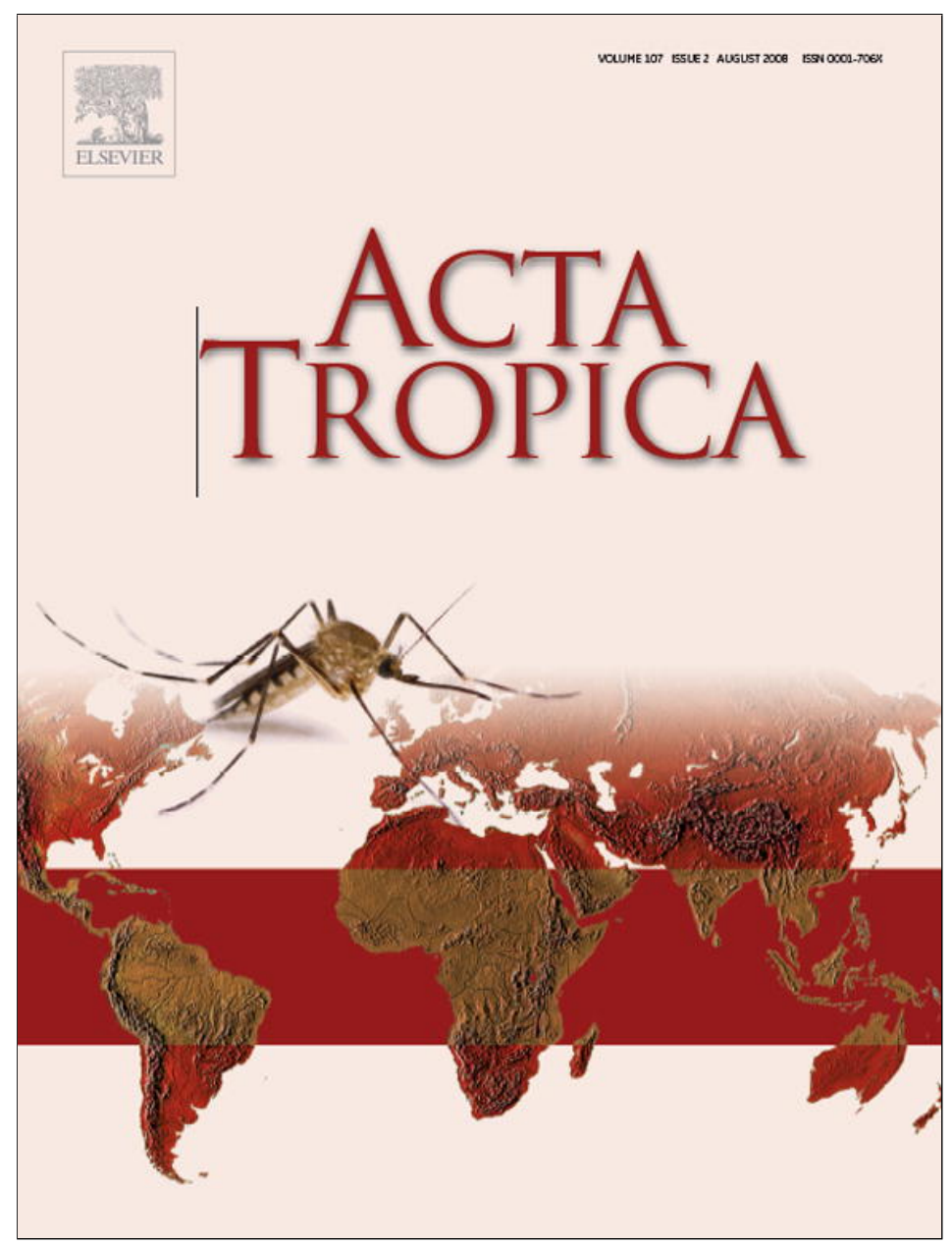

This article appeared in a journal published by Elsevier. The attached copy is furnished to the author for internal non-commercial research and education use, including for instruction at the authors institution and sharing with colleagues.

Other uses, including reproduction and distribution, or selling or licensing copies, or posting to personal, institutional or third party websites are prohibited.

In most cases authors are permitted to post their version of the article (e.g. in Word or Tex form) to their personal website or institutional repository. Authors requiring further information regarding Elsevier's archiving and manuscript policies are encouraged to visit:

http://www.elsevier.com/copyright 


\title{
Flow cytometric enumeration of parasitaemia and haematologic changes in trypanosoma-infected mice
}

\author{
Nicolas Antoine-Moussiaux $^{\mathrm{a}, \mathrm{b}, *}$, Dirk Saerens $^{\mathrm{c}, \mathrm{d}}$, Daniel Desmecht $^{\mathrm{a}}$ \\ a Department of Animal Pathology, Faculty of Veterinary Medicine, Sart Tilman B43, University of Liège (ULg), Belgium \\ ${ }^{\mathrm{b}}$ Department of Animal Production, Faculty of Veterinary Medicine, Sart Tilman B43, University of Liège (ULg), Belgium \\ ${ }^{\mathrm{c}}$ Laboratory of Cellular and Molecular Immunology, Vrije Universiteit Brussel, Pleinlaan 2, B-1050 Brussels, Belgium \\ d Department of Molecular and Cellular Interactions (CMIM), Flanders Institute for Biotechnology (VIB) Pleinlaan 2, B-1050 Brussel, Belgium
}

\section{A R T I C L E I N F O}

\section{Article history:}

Received 30 January 2008

Received in revised form 9 May 2008

Accepted 16 May 2008

Available online 23 May 2008

\section{Keywords:}

Trypanosoma

Flow cytometry

C57BL/6

$\mathrm{BALB} / \mathrm{c}$

Parasitaemia

\begin{abstract}
A B S T R A C T
African trypanosomiasis is a severe parasitic disease affecting both man and livestock. It is crucial to expand our fundamental knowledge of the intimate interactions between trypanosomes and their vertebrate hosts in order to develop new and efficient control strategies. The mouse model of trypanosomiasis is the most popular for research purposes because of all the logistic advantages of using this species. Studies of any aspect of trypanosomiases in the mouse systematically require the quantification of some phenotypic traits which translate its degree of resistance/susceptibility to the disease, as blood cell counts. The present study presents a methodological approach combining everyday microsampling of tail blood and its analysis by flow cytometry. The technical options and conditions permitting a fast, reliable and reproducible daily quantification of erythrocyte, reticulocyte, leucocyte and trypanosome counts in the inoculated mouse were established. The protocol proposed allows the multiplication of blood samplings without being exposed to the time-consuming constraint of visual countings, without causing iatrogenic blood cell alterations in the mouse and without requiring specific anti-trypanosome antibodies.
\end{abstract}

(c) 2008 Elsevier B.V. All rights reserved.

\section{Introduction}

African trypanosomiases consist of a group of severe parasitic diseases that affect both man and livestock and represent a vital and economic threat to man in many prime areas of Africa, Asia and South America. These diseases are caused by flagellate protozoan parasites of the genus Trypanosoma, which enter the bloodstream via the bite of blood-feeding flies, escaping humoral immune response thanks to the fast turnover of their glycoproteic coat. One of the most crucial priority in this field is to expand our fundamental knowledge of the intimate interactions between trypanosomes and their vertebrate hosts in order to develop a first efficient vaccine and to replace longstanding therapeutic molecules that have become inefficient due to the dissemination of resistant strains. The mouse model of trypanosomiasis is the most popular for research purposes because of all the logistic advantages of using this species. Studies of any aspect of trypanosomiases in the mouse systematically require the quantification of some pheno-

\footnotetext{
* Corresponding author at: Department of Animal Pathology, Faculty of Veterinary Medicine, Sart Tilman B43, University of Liège (ULg), 4000 Liege, Belgium.

Tel.: +32 43664075 .

E-mail address: nantoine@ulg.ac.be (N. Antoine-Moussiaux).
}

typic traits which translate its degree of resistance/susceptibility to the disease, i.e. death or survival, evolution of body weight and blood cell counts. In this latter case, investigators face important limitations for repeated measurements in the same animal or multiple single measurement in a substantial number of animals. The first and principal is linked to the slowness and monotony of the reference enumeration method of parasitaemia, which consists of direct visual countings under the microscope (Lumsden et al., 1973). Beyond the total working time limitation, some might argue that the time spent between measurements in the case of large animal cohorts does not allow to consider them as really matched in the case of a rapid changing phenomenon such as trypanosome blood loads. The second has a biologic nature: as the mouse blood volume is small, it is difficult to sample it more than once without contributing to the worsening of the disease. As classic trypanosome enumeration method allow working on such small blood quantities, any new proposed method will have to meet this central criteria for further use in the mouse model. Because of these constraints, the dynamics of blood parasite and blood cells changes is not accessible, whereas it could reveal key informations on host-pathogen interactions. Prompted by three pioneering studies (Martin et al., 1972; Jackson et al., 1977; Mills and Valli, 1978), a methodological approach combining everyday microsampling of tail blood and its analysis by flow cytometry was developed. The technical 
options and conditions permitting a fast, reliable and reproducible daily quantification of erythrocyte, reticulocyte, leucocyte and trypanosome counts in the inoculated mouse were established. The protocol proposed allows the multiplication of blood samplings without being exposed to the time-consuming constraint of visual countings, without causing iatrogenic blood cell alterations in the mouse and without requiring specific anti-trypanosome antibodies.

\section{Materials and methods}

\subsection{Design}

Peripheral blood of ten mice from each of two different inbred strains (BALB/CJ and C57BL/6J) was collected daily for 8 (BALB/CJ) or 39 (C57BL/6J) days and analyzed for haematological and parasitological values determinations by flow cytometry. On day 9, C57BL/6 mice were inoculated intraperitoneally with a Trypanosoma evansi isolate. Accuracy of flow cytometric trypanosome counts was assessed by comparing the values obtained with those generated by visual countings using the Neubauer's chamber. In selected C57BL/6J mice, 10 consecutive blood samplings were also drawn the same day to assess intra-day reproducibility.

\subsection{Mice, parasite, and inoculation}

The experiments were conducted with specific pathogen-free 16-week-old female mice obtained from Charles River Laboratories. Housing, inoculation, sampling and euthanasia procedures complied with National Institutes of Health guidelines, and the experimental protocol was approved by the Bioethics Committee of the University of Liège. The T. evansi strain (ITMAS code 220404A, generously supplied by P. Büscher, Institute of Tropical Medicine, Antwerp, Belgium) had been isolated in 1997 from a diseased camel (Zagora, Morocco) and was preserved since in liquid nitrogen. The isolate was first passed once in immunodepressed BALB/cJ mice (cyclophosphamide, $200 \mathrm{mg} / \mathrm{kg}$ of body weight) to produce the stock solution. The stock solution was then diluted in Phosphate Saline Glucose containing 5\% DMSO (final concentration), separated in aliquots, and stored in liquid nitrogen (final trypanosome concentration: $10^{7} \mathrm{ml}^{-1}$ ). The inoculation procedure consisted in the intraperitoneal injection of $100 \mu \mathrm{l}$ of this flagellate suspension adjusted at $10^{6}$ viable trypanosomes per $\mathrm{ml}$.

\subsection{Blood sampling and processing}

A volume of $1-3 \mu \mathrm{l}$ tail blood was collected by capillarity with a heparinized $75 \mu \mathrm{l}$ micro-haematocrit tube (Hirschmann Labogeräte, Germany). One microlitre was diluted in $900 \mu$ l Dulbecco's phosphate buffered saline (PBS). Two microlitres of a $0.33-\mathrm{mg} / \mathrm{ml}$ solution of allophycocyanin (APC)-conjugated affinitypurified rat IgG2b directed towards murine TER-119 erythrocyte glycophorin-like receptor (eBiosciences Inc., USA) were then added and the cell suspensions were incubated at room temperature in the dark for $20 \mathrm{~min}$. After washings, cell suspensions were fixated by incorporating $95 \mu \mathrm{l}$ of PBS-formaldehyde (10\%) and stained further by adding $2 \mu \mathrm{l}$ of a $1 \mathrm{mg} / \mathrm{ml}$ propidium iodide (PI) solution (Invitrogen, USA) for nucleic acids labelling. The cell suspensions were then stored at $4{ }^{\circ} \mathrm{C}$ for $48 \mathrm{~h}$ before analysis. Blood was thus diluted to $10^{-3}$ before quantitation attempts were started.

\subsection{Flow cytometry}

Data acquisition was performed with a BD FACSCanto ${ }^{\mathrm{TM}}$ flow cytometer. PI and APC were detected separately using 488 and
$633 \mathrm{~nm}$ excitation with $670 \mathrm{~nm}$ (FL3) low-pass and 660/20 nm (FL5) bandpass emission filters, respectively. Reproducibility of the fluorescence readings had been established before the measurements using fluorophore-conjugated polymethylmethacrylate microspheres (BD FACS 7-color Setup beads). The sample flow rate was set at medium rate $(1 \mu \mathrm{l} / \mathrm{s})$. For one single analysis, the scattered and fluorescent lights emitted by 100,000 cells were picked up, which roughly required $10 \mathrm{~s}$ per sample. The data were then analyzed using the BD FacsDIVA ${ }^{\mathrm{TM}}$ software. Absolute counts were retrieved by multiplying the number of events detected in each subpopulation of events by the volume of the suspension analyzed by the cytometer and by normalizing the result obtained to $1 \mathrm{ml}$ of mouse blood (taking the dilution factor of the blood into consideration). The volume of the cell suspension analyzed is the product of the duration of the analysis(s) by the sample flow rate $(1 \mu \mathrm{l} / \mathrm{s})$.

\subsection{Identification of trypanosomes}

Demonstration that the extra cell population detected in dot plots from inoculated mice (see after) was made of trypanosomes was obtained by three different ways. The extra population was first sorted and the morphology of the sorted events was ascertained by direct visual inspection under the microscope. Second, the dot plot signature of a trypanosome suspension purified by passing infected blood on a DEAE-cellulose column was compared to that of the extra population detected in infected blood. Third, blood samples from parasitaemic mice were processed as described except that the erythrocytic glycophorin staining step was replaced by a specific labelling of trypanosomes, which allowed a direct vizualisation of the parasites on the dot plots. For the staining, $10 \mu \mathrm{l}$ of a $200-\mu \mathrm{g} / \mathrm{ml}$ solution of the Alexa-488-conjugated NbTRYP14 Nanobody directed towards non-VSG surface epitopes and known to react with T. evansi (Saerens et al., 2008) were added and the blood suspensions were incubated at room temperature in the dark for $30 \mathrm{~min}$.

\subsection{Quality assessment of flow cytometric trypanosome counts}

Accuracy of flow cytometric trypanosome counts was first assessed by comparing them with counts obtained from the same blood sample by the reference technique using direct visual countings in the Neubauer's chamber (Lumsden et al., 1973). To do this, 10 blood samples were drawn at $30 \mathrm{~s}$ interval from two mice for four consecutive days, during the expected exponential rise of parasitaemia, as determined by a preliminary follow-up of a set of mice with the rapid matching method. The resulting 80 blood samples, thus supposed to cover a wide range of trypanosome counts, were then analyzed by both methods. The same set of data yielded eight coefficients of variation measuring the intra-day inter-sampling reproducibility, i.e. an estimate of the total variability generated by the whole process (tail blood sampling, blood dilution, cells fixation and labelling, flow cytometry and graphical definition of the population being counted). Intra-day intra-sampling inter-analysis reproducibility was evaluated by determining the coefficient of variation calculated from 10 successive analyses of 6 blood samples with parasitemias ranging between $10^{6}$ and $10^{9}$ trypanosomes per millilitre of blood. This coefficient is an estimate of the variability generated by the graphic selection of the trypanosome-like population. Effect of the duration of sample conservation in the fridge (after labelling) on trypanosome, erythrocyte, reticulocyte and leucocyte counts was tested by comparing the results obtained from a set of 10 blood samples of which an aliquot was extracted and analyzed 1, 2, 6 and 20 days after processing. Sensitivity of flow cytometric trypanosome countings was evaluated by analyzing successive dilutions of a blood sample containing about $10^{7}$ 

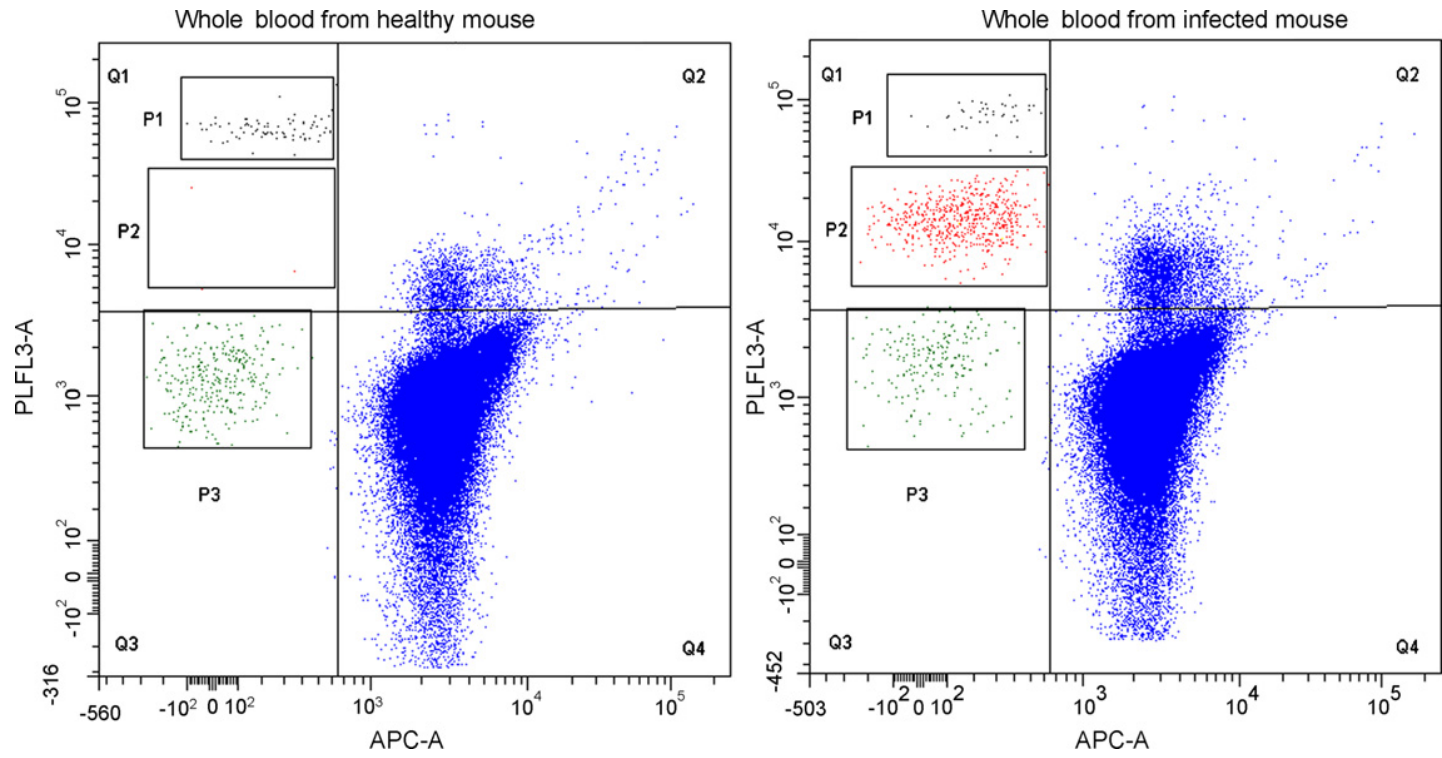

Fig. 1. Propidium iodide (nuclear staining) vs. allophycocyanin (erythrocyte staining) fluorescence intensity dot plots from flow cytometric analysis of healthy (left) and inoculated (parasitaemic, right) mouse whole blood. Q quadrant; P, graphically selected cell populations; Q1 contains the leukocyte (P1) and the trypanosome populations (P2), the latter being absent in the healthy mouse (left); Q2 and Q4 contain immature (nucleated) and mature erythrocytic populations, respectively and events falling in Q3 correspond to thrombocytes, cellular and non-cellular debris.

parasites per millilitre. In order to keep all background conditions constant, the labelled parasitaemic blood was diluted by similarly labelled blood drawn from a healthy mouse. Effect of daily sampling of $1-3 \mu$ l of tail blood on erythropoiesis was assessed by monitoring reticulocyte counts for 8 consecutive days in 10 healthy mice.

The results are presented as means \pm standard deviation. Statistical analyses addressing the reproducibility tests and the effect of daily blood sampling on erythropoiesis were made through an analysis of variance using the $\mathrm{SAS}^{\circledR}$ software (version 8.2 ; $\mathrm{glm}$ procedure). Duncan's ranking method was used as a post hoc test (SAS Institute Inc., USA).

\section{Results}

\subsection{Graphical definition of cell populations}

For each set of blood samples that were processed simultaneously, the 100,000 events detected from a control mouse were first retrieved in the form of a propidium iodide/allophycocyanin fluorescence intensity dot plot (Fig. 1). By doing so, four quadrants (Q1-4) were drawn using the threshold settings identified from prior autofluorescence studies, Q1-2 gathering nucleic acids-containing glycophorin-negative (Q1) and -positive (Q2, reticulocytes) events and Q3-4 nucleic acids-negative glycophorinnegative (Q3, cell debris) and -positive (Q4, erythrocytes) events. Within the glycophorin-negative quadrants (Q1 and Q3), two nonadjacent populations of events were easily outlined (areas P1 and P3), always leaving a well-demarcated empty area (area P2). Gating on the same dot-plot and using these preestablished quadrant/area definitions, the data of infected mice were then retrieved (Fig. 1). The general picture obtained resembled that typical of control mice, except that a new population of events appeared into the formerly empty P2 area (nucleic acids-positive and glycophorinnegative).

In the side scatter/forward scatter (SSC/FSC) representation of the $\mathrm{P} 1$ populations, three cell subsets were readily discriminated on the basis of their high-SSC (P1a), low-SSC/low-FSH (P1b) and lowSSC/high-FSH (P1C) (Fig. 2). By using specific markers, these cell subsets were shown to gather polymorphonuclear leucocytes (P1a), lymphocytes (P1b) and a mixed population containing monocytes and plasmocytes (data not shown). When the SSC/propidium iodide dot plot of the P2 populations of events were constructed, they always revealed a highly homogeneous population with respect to cell complexity (Fig. 3). This view was used to improve the graphical selection of the $\mathrm{P} 2$ population by delimitating the polygon enclosing the cohort of SSC-matched cells. Histological assessment of sorted P2 cells fixed on slides from a set of infected blood samples revealed that $96.2 \pm 1.4 \%$ of events falling into the P2 area were trypanosomes. Upon analysis of the purified trypanosome suspension, $>99 \%$ detected events fell in the aforedefined P2 area. Finally, 99\% of events gathered in the $\mathrm{P} 2$ area were simultaneously labelled with

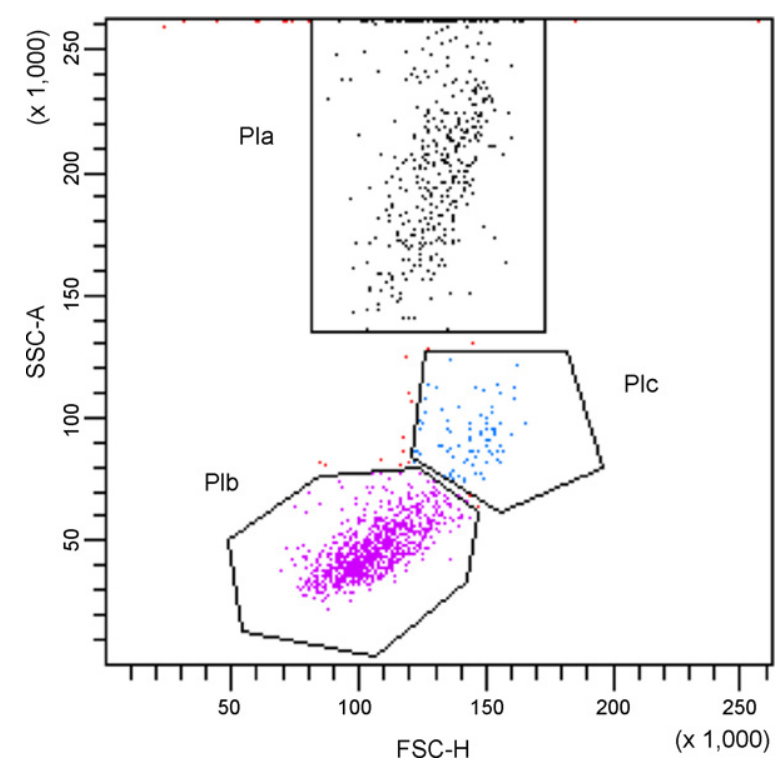

Fig. 2. Side (SSC) vs. forward (FSC) scatter dot plot of the P1 population (leukocytes, see Fig. 1) from mouse whole blood. SSC and FSC vary according to cell granularity and size, respectively. P1a, granulocytes (high granularity); P1b, agranulocytic population 1 (low SSC and low FSC, corresponds to lymphocytes) and P1c, agranulocytic population 2 (low SSC and high FSC, corresponds to mono-/plasmocytes). 


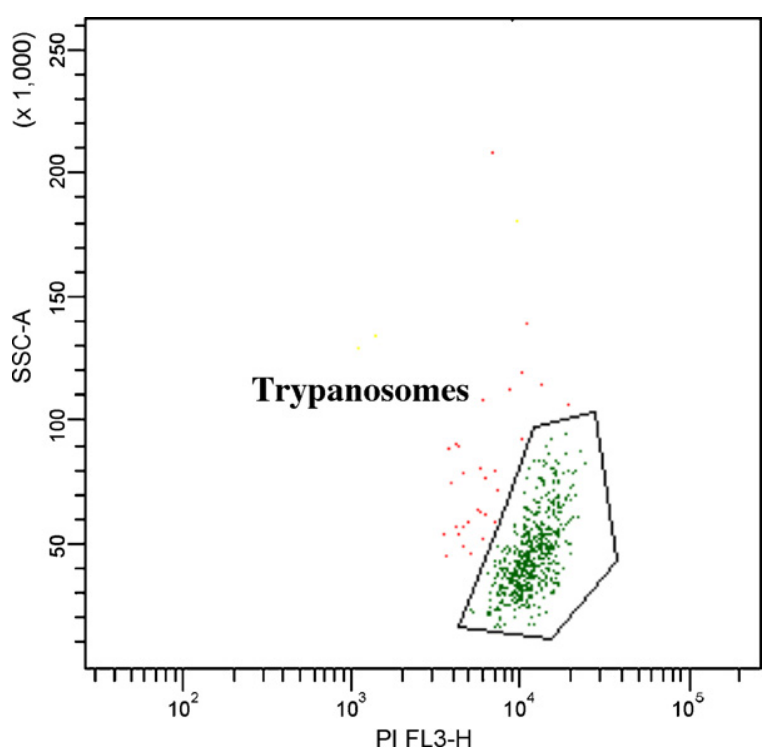

Fig. 3. Side scatter vs. propidium iodide fluorescence dot plot of the putative trypanosome population. The P2 population graphically selected on the PI/APC dot plot (Fig. 1) was graphically restricted to the compact population shown here, which was used for counting.

the Nanobody directed towards non-VSG trypanosomal surface epitopes (Fig. 4).

\subsection{Quality assessment of flow cytometric trypanosome counts}

Flow cytometric trypanosome counts were first compared to the counts yielded by the reference procedure using the Neubauer's chamber (Table 1). Statistical analysis ( $t$-test) did not reveal significant differences between parasitaemias $(p>0.5)$. With respect to intra-day inter-sampling reproducibility, observed coefficients of variation (CV) decreased as parasitaemia increased as expected from Poisson's law (Table 1). They ranged from 11.9\% (high parasitaemia) to $25.7 \%$ (low) and from $10.2 \%$ to $21.6 \%$ for Neubauer's and flow cytometry countings, respectively, being systematically lower by flow cytometry. Coefficients of variation quantifying intraday intra-sample inter-analysis reproducibility are gathered in Table 2 . They ranged between $12.9 \%$ and $2.6 \%$ for low and high parasite loads, respectively, which is $1-2 \%$ above the expected values according to Poisson's law. Within-sample CVs abrupty rose for parasitaemias below $5 \times 10^{5}$ trypanosomes per millilitre of blood, suggesting the detection threshold should be set there. Keeping the labelled samples at $4{ }^{\circ} \mathrm{C}$ before flow cytometric analysis resulted
Table 1

Comparison between parasitemias yielded by flow cytometry and hemocytometry (Neubauer) countings

\begin{tabular}{|c|c|c|c|c|c|c|}
\hline \multirow{2}{*}{$\begin{array}{l}\text { Parasite } \\
\text { load }\end{array}$} & \multirow[t]{2}{*}{ Mouse } & \multicolumn{2}{|l|}{ Neubauer } & \multicolumn{2}{|c|}{ Flow cytometry } & \multirow{2}{*}{$\begin{array}{l}\text { Theoretical } \\
\text { CV }\end{array}$} \\
\hline & & Mean & CV (\%) & Mean & CV (\%) & \\
\hline \multirow[t]{2}{*}{$10^{6}$} & A & $3.42 \times 10^{6}$ & 25.69 & $3.25 \times 10^{6}$ & 21.62 & 16.57 \\
\hline & B & $5.84 \times 10^{6}$ & 24.05 & $5.22 \times 10^{6}$ & 20.73 & 13.10 \\
\hline \multirow[t]{2}{*}{$10^{7}$} & A & $4.69 \times 10^{7}$ & 15.63 & $4.72 \times 10^{7}$ & 13.55 & 4.69 \\
\hline & B & $7.37 \times 10^{7}$ & 22.23 & $6.49 \times 10^{7}$ & 13.84 & 3.92 \\
\hline \multirow[t]{2}{*}{$10^{8}$} & A & $3.35 \times 10^{8}$ & 14.08 & $3.23 \times 10^{8}$ & 10.14 & 1.94 \\
\hline & B & $6.55 \times 10^{8}$ & 13.60 & $6.13 \times 10^{8}$ & 9.88 & 1.27 \\
\hline \multirow[t]{2}{*}{$10^{9}$} & A & $1.06 \times 10^{9}$ & 10.71 & $9.16 \times 10^{8}$ & 10.55 & 1.02 \\
\hline & B & $1.89 \times 10^{9}$ & 11.94 & $1.82 \times 10^{9}$ & 10.25 & 0.50 \\
\hline
\end{tabular}

Mean, least square mean (parasites per ml blood); $\mathrm{CV}$, coefficient of variation; Theoretical CV calculated according to Poisson's law, by the formula CV $=100 / N^{1 / 2}$, $N=$ mean number of counted events on the 100,000 analyzed. For each parasitaemia level, 10 samples were analyzed for each of two mice.

Table 2

Intra-sample inter-analysis reproducibility of trypanosome counts yielded by flow cytometry

\begin{tabular}{lcc}
\hline Mean & Observed CV $(\%)$ & Theoretical CV (\%) \\
\hline $4.60 \times 10^{6}$ & 12.95 & 11.65 \\
$1.21 \times 10^{7}$ & 8.43 & 7.38 \\
$4.00 \times 10^{7}$ & 5.30 & 4.15 \\
$1.24 \times 10^{8}$ & 3.30 & 2.29 \\
$2.72 \times 10^{8}$ & 2.55 & 1.17 \\
$1.74 \times 10^{9}$ & 2.63 & 0.50 \\
\hline
\end{tabular}

Mean, arithmetic mean (parasites per ml blood); CV, coefficient of variation; theoretical CV calculated according to Poisson's law, by the formula $C V=100 / N^{1 / 2}, N=$ mean number of counted events on the 100,000 analyzed.

in significantly different counts of erythrocytes, reticulocytes and leucocytes (Table 3 ). Trypanosome numbers remained stable up to $48 \mathrm{~h}$ and decreased after. Staining of leucocytes (propidium iodide) improved up to $48 \mathrm{~h}$ and remained constant after up to 20 days.

\subsection{Haematological values in control mice}

Circulating erythrocyte and leucocyte counts averaged $6.6 \pm 0.5 \times 10^{9}$ cells and $6.0 \pm 0.8 \times 10^{6}$ cells per millilitre of blood in C57BL/6J and $7.1 \pm 0.6 \times 10^{9}$ cells and $4.9 \pm 0.9 \times 10^{6}$ cells per millilitre in BALB/cJ mice, respectively. Granulocyte/agranulocyte ratios and reticulocyte percentages were $0.24 \pm 0.03$ and $3.72 \pm 0.63$ (C57BL/6J) or $0.42 \pm 0.06$ and $2.60 \pm 0.26(\mathrm{BALB} / \mathrm{cJ})$. Mean erythrocytic FSC values were $630 \pm 5 \times 10^{2}$ and $645 \pm 4 \times 10^{2}$. As
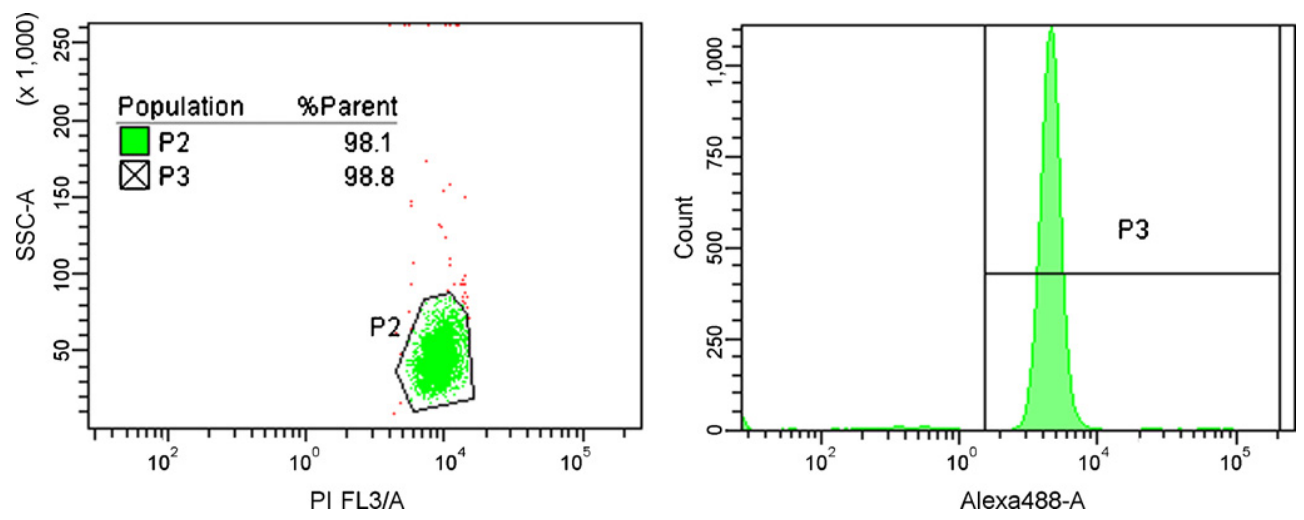

Fig. 4. The P2 cell population is homogeneously stained by a trypanosome-specific marker. SSC, side scatter; PI, propidium iodide; Alexa488, fluorescent label tagging trypanosome-specific Nanobody NbTRYP14. 
Table 3

Effect of samples conservation on blood cell counts

\begin{tabular}{lllll}
\hline Day p.s. & Trypanosomes & Leucocytes & Erythrocytes & Reticulocytes \\
\hline 1 & $1.82 \times 10^{9} \mathrm{a}$ & $2.24 \times 10^{6} \mathrm{~b}$ & $2.71 \times 10^{9} \mathrm{a}$ & $7.75 \times 10^{7} \mathrm{bc}$ \\
2 & $1.76 \times 10^{9} \mathrm{ab}$ & $3.81 \times 10^{6} \mathrm{a}$ & $2.57 \times 10^{9} \mathrm{~b}$ & $7.44 \times 10^{7} \mathrm{c}$ \\
6 & $1.68 \times 10^{9} \mathrm{~b}$ & $3.87 \times 10^{6} \mathrm{a}$ & $2.50 \times 10^{9} \mathrm{~b}$ & $8.79 \times 10^{7} \mathrm{~b}$ \\
20 & $1.49 \times 10^{9} \mathrm{c}$ & $3.83 \times 10^{6} \mathrm{a}$ & $2.23 \times 10^{9} \mathrm{c}$ & $11.83 \times 10^{7} \mathrm{a}$ \\
Trend & Decrease & Stable & Decrease & Increase
\end{tabular}

Values given are least square means for each cell population at days 1, 2, 6 and 20 for a series of 10 samples. A same letter (a, b or c) is attributed to values that are not statistically different.

reticulocyte counts remained constant between $3 \%$ and $4 \%$ of total erythrocyte number $(p>0.5)$ throughout the observation period, despite daily 1-3 $\mu$ l tail blood samplings, it is assumed that the procedure did not significantly impact erythropoiesis.

\subsection{Haematological values in principal mice}

Typical dynamic day-by-day evolution of parasitaemia, erythrocyte, reticulocyte, granulocyte and agranulocyte counts in a representative mouse is presented (Fig. 5). The characteristic recurrent peaking of parasitaemia is readily detected. Interestingly, the procedure also highlighted a tidal pattern in all other blood cell populations, the detailed description of which is beyond the scope of the current study. All C57BL/6J mice showed similar dynamics. Parasitaemia topped the detection threshold on and after the third or the fourth day after inoculation in all mice, peaked for the first time on day 5 or 6 after inoculation and never exceeded $10^{9}$ parasites per millilitre throughout the study (30 days).
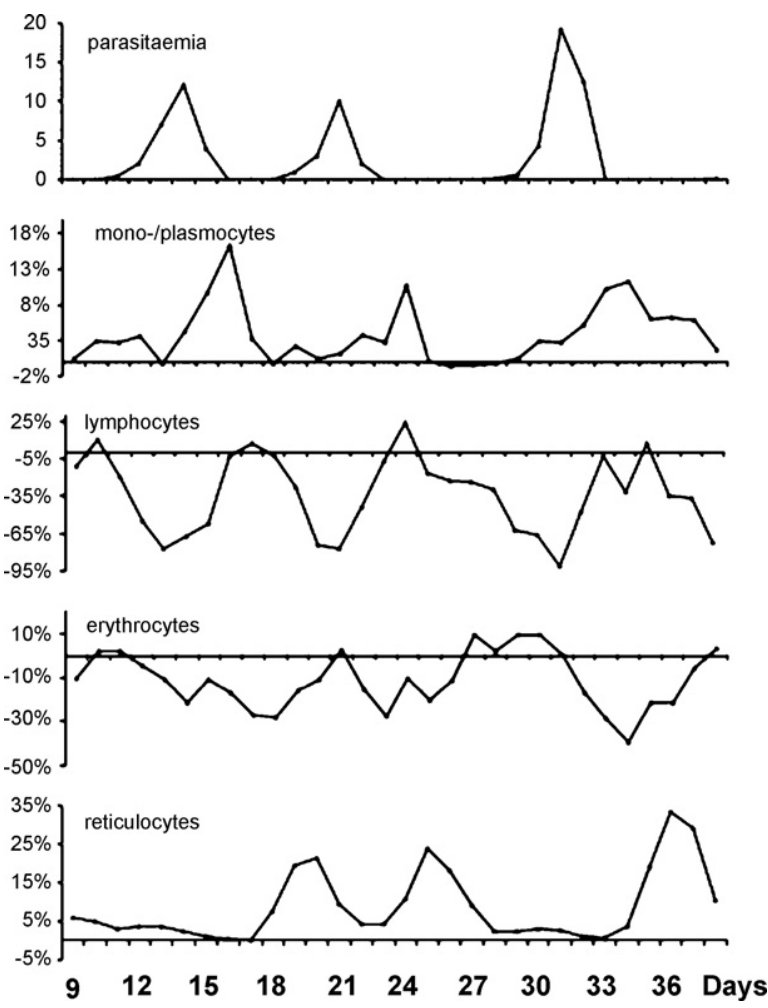

Fig. 5. Typical day-by-day follow-up of peripheral blood values in a mouse infected by $T$. evansi. The cyclic nature of parasitaemia (number $\times 10^{7}$ ) accompanying trypanosomiasis is clearly observed. A recurrent pattern is also observed for leukocytes, erythrocytes and reticulocytes counts (all expressed as percentages of deviation from individual preinoculation values).

\section{Discussion}

The reference procedure for enumeration of trypanosomes consists in a direct visual counting method in which numbers of motile trypanosomes are estimated using a Neubauer haemocytometer chamber (Lumsden et al., 1973). Slowness and monotony are two practical drawbacks of the method in the laboratory, which prompted us to examine the possibility that enumeration could be automatized by using flow cytometry. As trypanosomes constantly change their antigenic coat, their specific labelling was not the preferred option at the beginning because high-quality antibodies are not always available. In preliminary experiments in which buffy coats were analyzed, it was found that trypanosomes typically accumulated less propidium iodide per cell than leucocytes, but more than platelets or cell debris, which suggested that an antibody-less strategy could be developed. As it was impossible to discriminate trypanosomes from reticulocytes by only using propidium iodide and because simultaneous haematologic values were highly desirable, we opted for a double labelling strategy in which nucleic acids (propidium iodide) and the erythrocyte-specific glycophorin antigen (allophycocyanin) are tagged. As automatic countings were intended, the critical point became that of graphic selection of cell populations on dot plots, in which the subjectivity of the operator could theoretically hamper reproducibility. In the context of standard trypanosome enumeration, however, direct visual counting also implies some subjectivity. When graphically selected trypanosome-like cells were inspected under the microscope, $96.2 \pm 1.4 \%$ were indeed trypanosomes. Enumeration of trypanosomes in eighty 1-3 $\mu$ l blood samples covering a wide range of parasitaemias by direct counting in a Neubauer chamber and by flow cytometry yielded similar results (Table 1 ), which suggest that the time-consuming direct counting could be advantageously replaced by flow cytometry with no loss of accuracy. Moreover, flow cytometric counting yielded slightly lower CVs between successive samples drawn at the same time point. Setting the maximum intra-day intra-sample inter-analysis CV at $15 \%$, we found that the flow cytometric detection threshold is about $5 \times 10^{5}$ flagellates per millilitre, which is roughly similar to that claimed for the direct visual counting method (Lumsden et al., 1973). According to Poisson's law, this threshold will automatically diminish if more events (larger blood volume) are analyzed. This is obviously possible with the flow cytometer ( $\sim 10 \mathrm{~s}$. for acquiring the characteristics of 100,000 cells) but is unrealistic with the direct counting method. The ideal delay between labelling and analysis is $48 \mathrm{~h}$. Below, complete staining of leucocytes is not optimal and after there is a progressive loss of erythrocytes, reticulocytes and trypanosomes, probably due to autolysis. Removal of up to $1 \%$ of total blood volume daily over time (thus $12-16 \mu \mathrm{l}$ for a 20 -g mouse) has been claimed to be feasible without affecting the mouse blood parameters (Hoff, 2000). The 1-3 $\mu$ l volumes drawn daily here were far below this standard and were proven to be compatible with constant reticulocyte counts, suggesting that changes in erythropoiesis detected by such everyday samplings are attributable to biologic causes rather than to the protocol itself. The protocol thus provides a tool to catch short-lived pathological effects affecting blood cells, which could dramatically improve knowledge on host-pathogen interactions in view of the rapidly fluctuating parasitaemia in trypanosomainfected mice.

Comparison of haematologic values gathered in this study from non-inoculated mice with those available in the Mouse Phenome Database (www.jax.org/phenome) is presented (Table 4). In a general way, the archived absolute values and differences between C57BL/6J and BALB/CJ strains were similarly retrieved from the present flow cytometric measurements. Interestingly the higher mean erythrocytic FSC in BALB/cJ found in this study paral- 
Table 4

Comparison of haematological values in $\mathrm{BALB} / \mathrm{cJ}$ and $\mathrm{C} 57 \mathrm{Bl} / 6 \mathrm{~J}$ mice obtained by flow cytometry in this study with reference values from The Jackson Laboratory

\begin{tabular}{|c|c|c|c|c|}
\hline \multirow[t]{2}{*}{ Haematological values } & \multicolumn{2}{|l|}{ This study } & \multicolumn{2}{|c|}{ Mouse Phenome Database (www.jax.org) } \\
\hline & $\mathrm{BALB} / \mathrm{cJ}$ & $\mathrm{C} 57 \mathrm{Bl} / 6 \mathrm{~J}$ & $\mathrm{BALB} / \mathrm{cJ}$ & C57Bl/6J \\
\hline Erythrocytes* $\left(\times 10^{9} \mathrm{ml}^{-1}\right)$ & $7.1(0.6)$ & $6.6(0.5)$ & $9.4(0.3)$ & $10.2(0.3)$ \\
\hline Reticulocytes (\%) & $2.6(0.3)$ & $3.7(0.6)$ & $2.2(0.1)$ & $3.3(0.8)$ \\
\hline Leucocytes* $\left(\times 10^{6} \mathrm{ml}^{-1}\right)$ & $4.9(0.9)$ & $6.0(0.8)$ & $4.8(1.2)$ & $5.9(1.1)$ \\
\hline Granulocytes* (PMN) (\%) & $29.8(4.9)$ & $19.5(3.1)$ & $14.8(3.1)$ & $7.7(1.8)$ \\
\hline Agranulocytes* (Lympho) (\%) & $70.2(3.3)$ & $80.5(2.3)$ & $80.0(3.7)$ & $87.9(2.2)$ \\
\hline Erythrocytes size* (FSC/MCV) & $64500(400)$ & $63000(500)$ & $47.2(0.8) \mathrm{fL}$ & $45.1(0.5) \mathrm{fL}$ \\
\hline
\end{tabular}

This table compares haemotological values obtained in this study to those available on the Mouse Phenome Database (MPD) from The Jackson Laboratory. When available, MPD's values were taken for age groups similar to our study (marked with *). The global granulocytes percentage from our study is here compared to polymorphonuclear percentage from MPD, this cell type being numerously largely dominant among granulocytes. Similarly, the agranulocytes percentage is compared to lymphocytes percentage from MPD, monocytes representing less than $3 \%$ of total agranulocytes. The FSC (forward scatter) is a morphological parameter which is typical for flow cytometry and gives information about cell size. It has thus been here compared to the mean cell volume (MCV) available from MPD, which is expressed in femtolitres.

lels the higher mean corpuscular volume in BALB/cJ archived in the database. Therefore, erythrocytic FSC possibly could objectify pathological alterations of red blood cells during diseases. In trypanosome-infected mice, recurrent parasitic waves were nicely detected. Follow-up of erythrocytic count revealed recurrences of haemolytic crises followed by recoveries concomitant with episodes of reticulocytosis peaking at $30-40 \%$, then going back below the expected threshold of $5 \%$. Still considering the day-to-day dynamics of the disease, it is noteworthy that peaks of parasitaemia also roughly correspond to nadirs in leucocyte counts suggesting a relationship.

A new cheap, fast, accurate and reproducible trypanosome enumeration method using flow cytometry is described. Combined with daily microsampling of tail blood, it simultaneously provides a close follow-up of parasitic and haematologic values in the mouse, which offer the possibility to bring new insights in the pathogenesis of trypanosomiases. The method is equally successful to detect and count other trypanosomes such as T. brucei, T. congolense and T. vivax (data not shown) without the need of specific antibodies. As such, it can be implemented for a wide array of trypanosome species whenever fast, recurrent or mass monitoring of murine infection is desirable.

\section{Acknowledgements}

The authors are grateful to Prof. P. Büscher from the Institute of Tropical Medicine (Antwerp, Belgium) for the gift of the T. evansi isolate and for his expertise, help and enthusiasm. Many thanks are also due to Prof. A. Vanderplasschen and Dr. B. Dewals for free access to the facilities of the faculty laboratory of immunology, their precious help and challenging discussions. D.S. is Postdoctoral Fellow of the Research Foundation, Flanders (FWO).

\section{References}

Hoff, J., 2000. Methods of blood collection in the mouse. Lab. Animal 29, 4753.

Jackson, P.R., Winkler, D.G., Kimzey, S.L., Fisher, F.M., 1977. Cytofluorograf detection of Plasmodium yoelii, Trypanosoma gambiense, and Trypanosoma equiperdum by laser excited fluorescence of stained rodent blood. J. Parasitol. 63, 593598.

Lumsden, W.H.R., Herbert, W.J., McNeillage, G.J.C., 1973. Techniques with trypanosomes. Churchill-Livingstone, Edinburgh, pp. 95

Martin, L.K., Sadun, E.H., Ingram, G.D., 1972. Experimental infections with African trypanosomes. I. Quantitation of Trypanosoma rhodesiense by electronic particle counting. Am. J. Trop. Med. Hyg. 21, 880-884.

Mills, J.N., Valli, V.E., 1978. A cytofluorometric method of counting trypanosomes. Tropenmed. Parasitol. 29, 95-100.

Mouse Phenome Database: haematology measurements involving C57BL/6] [online] URL: http://aretha.jax.org/pub-cgi/phenome/mpdcgi?rtn=views/scanstats\& mode=strainheading\&stocknum $=000664 \&$ reqheading=hematology.

Mouse Phenome Database: haematology measurements involving BALB/cJ [online] URL: http://aretha.jax.org/pub-cgi/phenome/mpdcgi?rtn=views/scanstats\& mode $=$ strainheading\&strainid $=5$ \&reqheading $=$ hematology

Saerens, D., Stijlemans, B., Baral, T.N., NguyenThi, G.T., Wernery, U., Magez, S., DeBaetselier, P., Muyldermans, S., Conrath, K., 2008. Parallel selection of multiple anti-infectome Nanobodies without access to purified antigens. J. Immunol Methods 329, 138-150. 